\title{
Patenting and licensing in genetic testing
}

\author{
Recommendations of the European Society of Human Genetics
}

\section{S Aymé, G Matthijs and S Soini, on behalf of the ESHG Working Party on Patenting and Licensing}

The members of the Public and Professional Policy Committee (PPPC) and the Patenting and Licensing Committee (PLC) of ESHG who were involved in setting up these recommendations were Ségolène Aymé (Paris, France), Gert Matthijs (Leuven, Belgium), Violetta Anastasiadou (Nicosia, Cyprus), Fatmahan Atalar (Istanbul, Turkey), Suzanne Braga (Berne, Switzerland), John Burn (Newcastle upon Tyne, UK), JeanJacques Cassiman (Leuven, Belgium), Martina Cornel (Amsterdam, The Netherlands), Domenico Coviello (Milano, Italy), Gerry Evers-Kiebooms (Leuven, Belgium), Philippe Gorry (Bordeaux, France), Shirley Hodgson (London, UK), Helena Kääriäinen (Turku, Finland), György Kosztolányi (Pécs, Hungary), Ulf Kristoffersson (Lund, Sweden), Milan Macek Jr (Prague, Czech Republic), Christine Patch (London, UK), Jörg Schmidtke (Hannover, Germany), Jorge Sequeiros (Porto, Portugal), Dominique Stoppa-Lyonnet (Paris, France), Lisbeth Tranebjærg (Copenhagen, Denmark), Veronica van Heyningen (Edinburgh, UK) and Gert-Jan van Ommen (Leiden, The Netherlands).

Patents for inventions can be beneficial for society, if they drive innovation and promote progress. In most areas, the patenting system works satisfactorily. However, it must be recognized that in some instances it can also be problematic; this is the case in the field of genetics, and particularly in the area of genetic testing. As patents should serve their original purpose (promoting innovation through a fair reward system for the inventors), the European Society of Human Genetics (ESHG) suggests ways to improve the mechanisms that already form part of the patents system as a whole. In brief, the ESHG recommends limiting the breadth of the claims in genetic patents and, more practically, to reduce the number of patents by limiting the patentable subject matter, thereby improving the quality of the patents that will eventually be granted. There is also a suggestion to redefine the concept of utility in patent law, by taking account of downstream clinical experience. The ESHG sees no harm in the patenting of novel technical tools for genetic testing (eg PCR or chip technologies), as they can promote investment and still allow for invention around them. Many disputes between supporters of the patenting system and the public revolve around ethical issues. The European Patent Office should consider the benefit of having an ethics committee to consider issues of major interest, such as patents applied to genes. The problem of licensing should also be addressed. Practically, this means supporting the Organisation for Economic Co-operation and Development guidelines, which prescribe that licenses should be non-exclusive and easily obtainable, both in practical and in financial terms. To promote this, the practical exploration of alternative models for licensing, like patent pools and clearinghouses, is a prerequisite. To better track developments in this field, the establishment of a voluntary reporting system, whereby geneticists could report on any issues related to new and/or old patents or licenses in the light of service provision to patients, would be worthwhile. Finally, the ESHG is calling upon all stakeholders to start the process of developing a code of conduct for partners with patents, covering ethical aspects as well as smooth licensing arrangements. European Journal of Human Genetics (2008) 16, 405-411; doi:10.1038/sj.ejhg.5201929

Keywords: patents; licensing in genetics; DNA diagnostics; CHIP technology; European Patent Convention; OECD guidelines

Jointly developed by the European Journal of Human Genetics' Public and Professional Policy Committee (PPPC) and Patenting and Licensing Committee (PLC), assisted by multidisciplinary field experts. 


\section{Introduction}

The proliferation of patents on human genes has raised practical and ethical concerns, particularly in Europe. Typically, public opinion is against the patentability of human genes. The research community is uncertain about the impact on their research projects in the field. Healthcare professionals are worried about the impact of patents on the cost of genetic tests. Industries, especially small and medium enterprises, are troubled about the difficulties resulting from multiple licenses necessary to develop a new diagnostic kit or a new drug. In this response, the European Society of Human Genetics (ESHG) has established a dialogue between all stakeholders in this debate to review the facts and to identify ways to improve the current patenting and licensing system applied to the genetic testing field in order to better serve the public good in respect to current legislations. Jointly, the ESHG Public and Professional Policy Committee (PPPC) and the Patenting and Licensing Committee (PLC) organised a initiatory meeting on 29 November 2005 in Paris with four external experts, and an ESHG workshop on 13-14 November 2006 in Leuven, in which 18 experts were invited. Prior to the second workshop, each participant received a working paper developed by the PPPC and the PLC. This paper was revised after the workshop to take into account the points of view expressed by workshop participants. The resulting background document was posted on the ESHG website for discussion among the ESHG members. During the workshop, the participants were invited to provide recommendations they considered as crucial to improving the field. These recommendations were integrated with the background document, and considered by the ESHG in elaborating its own set of recommendations. The draft recommendations were presented to the Board of the ESHG in June 2007, and endorsed soon thereafter. They are presented below, as the ESHG Recommendations on Patenting and Licensing in Genetic Testing, and will be included, together with the full background document in a parallel supplementary issue of the European Journal of Human Genetics ${ }^{1}$.

A potential limitation in this process is that, in view of the current legal framework in Europe (with the European Patent Convention (EPC) and the Biotech Directive 98/44), it may be difficult for the current policies to be revisited. However, the fact that some Member States have been reluctant to transform the Biotech Directive into their national laws indicates that gene patenting is not readily accepted in Europe. Hence, Europe should remain open to the possibility of reopening the discussion on the patenting of genes and genomic data, especially when the debate has not been resolved at an international level.

Moreover, it is notably the body of licensing practices and especially the lack of guidelines for licensing - which is an integral part of the problem. On the one hand, a patent gives a monopoly to the patent holder, which may lead to widely objectioned exclusive licensing and unacceptably high costs of licensing fees. On the other hand, the modern tools that are being developed for genetic testing combine lots of data and often require access to a large number of patented genetic sequences. It is foreseeable that, in many cases, this will lead to complex situations and high costs, forcing manufacturers to refrain from the development and manufacturing of such tools - and hence slow down progress - or, even worse, to develop suboptimal diagnostic tools to avoid infringing on the patents. The ESHG welcomes the initiatives that were taken by the Organisation for Economic Co-operation and Development (OECD) to issue 'Guidelines for the Licensing of Genetic Inventions'. The implementation and use of such guidelines should be promoted further.

\section{Recommendations}

Patents for inventions can be beneficial for society, if they drive innovation and promote progress. In most areas, the patenting system works satisfactorily. However, it must be recognized that in some instances patents can also be problematic; this is the case in the field of genetics, and particularly in the area of genetic testing. For many geneticists, the patenting of genes remains questionable and even unacceptable from a principled point of view. Arguments include that the identification of a gene or a mutation, or a link between a genetic defect and a disease is not an invention but a discovery; and discoveries are not patentable according to the EPC. The argument that a cDNA does not exist in nature, and thus makes its sequence patentable, is often viewed as a legal twist that does not convince most geneticists. The patenting on genes and genomic sequences is intrinsically different from the patenting of methods, tools and technologies, because there is no possibility to 'invent around' a DNA or RNA (or cDNA) sequence. The patentability of nucleic acid sequences is generally defended on the basis that they are mere chemical structures; this viewpoint neglects the fact that genetic sequences also contain genetic information, which, in addition, is shared by all humans.

\section{The importance of genetic testing}

Genetic tests are an important component of health-care services, as they provide a way to establish difficult diagnoses and to detect persons at risk, before expressing the disease. The knowledge gained through genetic testing can be used to inform the concerned persons about the origin of their health problems, to provide them with an assessment of the risk for their relatives of developing similar problems, and the risk of passing on the condition to their children. Testing is also useful in planning clinical interventions that may benefit the concerned individuals, by attenuating or even efficiently treating their disease. It is therefore imperative that patients have access to tests that 
are valid, appropriate and affordable, within the context of the European health-care systems.

Once a link between a disease and a precise genetic defect has been established, the relevant diagnostic test can be relatively easily developed. Most laboratories use a combination of different methods: either kits or relatively simple, laboratory-developed ('home-brew') detection methods for the identification of one or a few mutations, or high-throughput scanning platforms or direct sequencing for the effective screening of the coding regions of a gene. The ease of development of genetic tests has allowed diagnostic laboratories to rapidly provide appropriate services. Drawbacks of this ease include the resulting diversity in quality of the services being offered and the potential lack of validation of the diagnostic methods.

Most genetic tests currently used are specific to a handful of genes (eg BRCA1 and BRCA2 for familial breast cancer), a single gene (eg CFTR for cystic fibrosis) or even a single mutation (eg the CAG repeat in the huntingtin gene for Huntington's disease). It is expected that this trend will change in several ways, whereby the complexity in the interpretation of the results and in intellectual property (IP) protection will increase. First, it is very likely that many tests in the future will be phenotype-based, exploring at once many potentially involved genes. In this context, the importance of patents on genes may increase further. This trend will play a role in, and may hamper, the development of gene chips. Second, tests will increasingly combine detection of several mutations with protein or metabolite measurements; thus combinations of patents will become the rule.

The patenting aspect is not the only issue of importance when considering the development of a new test. The assessment of its potential clinical use, the clinical validity and utility, is at least as important. Currently, the latter is not taken into account in the examination of the patent applications, except through the general utility requirement. Ideally, both should go hand in hand, whereby eventually, the clinical validity and utility would influence the patenting policy. This is a suggestion to redefine the concept of utility in patent law, by taking account of downstream clinical experience.

\section{The importance of patents as a reward system and as a promoter of progress and investment}

A patent grants the right to exclude others from exploiting an invention for 20 years from the date of the patent, usually the date of the filing. The patenting system is well established in many fields of technology and medicine. Patents that directly relate to the development of therapies are important for health care.

The prospect of IP protection is one of the factors that drive innovation and, certainly, translational research in the field of medical genetics. Patenting has become the norm in academic research institutions. It is the task of public research bodies to promote innovation, to transfer the results of publicly funded research to the public and to contribute to its wealth.

Hence, the ESHG does not propose a new model for IP protection in the field of genetics, but rather proposes to work with the existing patenting system and find complementary mechanisms facilitating access to patents, such as new licensing models.

The ESHG is aware of the fact that, if it proposes changes in the regulation of patenting and licensing in genetic testing, the whole patenting system would be affected. The latter would have an important economic impact. Any proposal for a change will thus have to be acceptable in other fields, to maintain consistency in the system.

As patents should serve their original purpose (promoting innovation through a fair reward system for the inventors), the ESHG suggests ways to improve the mechanisms that already form part of the patent system as a whole.

\section{Determine the size of the problem}

The influence of patents and (bad) licensing practices on the development and availability of genetic tests has been demonstrated through the BRCA case. Some have argued that the problem is negligible. The ESHG acknowledges that, thus far, only anecdotal or partial data are available. However, given that a considerable number of patents on disease genes and genetic tools have been granted, or are in the process of being granted, the ESHG considers that the issue cannot be ignored.

The genetics community and governments should survey the nature and scope of the alleged problem as it stands now, as well as how it may evolve in the future. There is a need to analyze the availability and accessibility of genetic tests in the public sector, and identify the responsibility of the patent system if tests that exist are not available or affordable.

The establishment of a voluntary reporting system (annually or case by case), whereby geneticists could report on any new and/or old patents or licenses perceived as damaging service to patients, would be worthwhile. This information would be shared with other stakeholders through all possible communication channels.

Interestingly, certain genetic inventions are patented in some countries, but not in others. Hence, the 'freedom to operate' is different in different countries. Even if this is a natural consequence of the fact that the patent system is in se a national matter, it further complicates the patent landscape, and may affect the genetic services for citizens in different countries, solely on the basis of the IP protection.

\section{Interact with all stakeholders}

Dialogue between patent owners, service providers, consumers and patients should be established to find a way to preserve the patent system without damaging the quality of care. 
Although patenting is an incentive for research and development, it may hinder complete access to products and may affect competition. As such, a balance must be set by the legislator to provide a framework for researchers to be rewarded for their inventions without preventing access to and affordability of genetic tests for patients. The ESHG is willing to take part in the discussions.

\section{The patentability of genetic inventions}

At present, DNA, RNA, genes and other components of the human genome are patentable under the EPC. Clearly, claims to DNA sequences have been held as acceptable by European Patent Office (EPO) on the condition that the basic patentability criteria are met. In most jurisdictions, these rules mean that the specification must enable the invention to be performed to the full extent of the monopoly claimed. If not, the claims must be narrowed to be valid, as the claims and the specification must be commensurate.

For this, the rules on sufficiency and support, that is Articles 83 and 84 of the EPC, require special attention.

It is clear that it is increasingly difficult to patent novel sequences, as applications are likely to require greater preparation and more biological data to support narrower claims. The ESHG sees no harm in the patenting of novel technical tools for genetic testing (eg PCR or chip technologies), as they can promote investment and still allow for invention around them.

\section{Avoid broad claims}

Irrespective of the size of the problem, the ESHG emphasizes that, due to the properties of genetic material, many claims that have been granted are overly broad. Broad claims are intrinsic in gene patents, due to the universal nature of gene translation and transcription and protein synthesis. For instance, patents on disease-related genes typically not only include claims on the nucleic acid sequence, but also on the protein and on the antibodies that can subsequently be generated, and on the animal models, even if they (still) have to be developed.

It is in the opinion of the ESHG that the EPO in the future may have to become yet more careful in assessing the scope of claims in genetic patents. It has been sufficiently illustrated that overly broad claims negatively impact development. The EPO could do this with the help of a scientific advisory committee. It could set up a (temporary) joint committee, including geneticists and patenting and licensing specialists, to analyse claims in the most recent patent applications to develop a better practice and to share expertise.

The possibility of a purpose-bound patent protection, as for example Germany, Italy and France have adopted it, is worth further examination, to limit unnecessary broad claims.

\section{Limit the patentable subject matter}

Patents on gene sequences will soon lose their importance, as most major disease genes were discovered over 10 years ago. Also, since the publication of the human genome, it is practically impossible to patent genetic sequences per se. Applicants may respond to the increasing tendency of patent offices to grant patents with narrower scope and more robust claims by filing patents claiming splice variants and SNPs.

Hence, one has to consider that the avalanche of patents has not necessarily ended. In fact, it is anticipated that the number of applications will remain high, and may even increase as a result of the growing research activities in China, India and Korea.

The relative excess of patents in the field may actually result from the high hopes that are created by the potential size of the market for genetic tests, especially for common disorders. It is the responsibility of geneticists to say what they foresee in that sector.

The ESHG proposes a relatively straightforward way to reduce the number of patents. It could be fairly easy to prohibit patenting of individual mutations in known disease genes, for example on the basis of an absence of novelty. A more important and socially acceptable ruling would be to consider that establishing a link between a disease and a genetic sequence or defect is merely a discovery and therefore not patentable, unless the identification of this link includes a real conceptual innovation. If the EPO would apply these criteria, and add them to their guidelines for patenting genetic inventions, it would prevent applicants from filing applications for patents that will not be granted anyway.

\section{Improve the quality of the granted patents}

Patent examination can also be improved, whereby all patent offices should be more selective in rewarding patent applications with greater quality and focus. The more stringent approach of European and Japanese patent examiners, as compared to their US colleagues, already demonstrates a trend in the right direction.

A scientific or professional body, for example the ESHG, should issue an annual statement on obviousness/nonobviousness to simplify the pursuit of a patent and provide incentives for reasonable licensing behaviour. This advisory body should also keep information on novel genetic mechanisms/methodologies for likely future patent or commercial protection up to date.

The ESHG is aware of the fact that the US Patent and Trademark Office is more liberal in its policy than the European and Japanese patent offices. This creates an uneven international situation, where patents are more easily granted in the United States as compared to Europe and Japan. The ESHG therefore welcomes and endorses the efforts of the Trilateral Organisation with respect to 
harmonising their approaches. International organisations, including the World Health Organization and the World Trade Organization, are, together with governments, responsible for alleviating differences that exist, and for warranting international equity in terms of IP protection.

\section{Reduce the backlog of patent examination}

It is the opinion of the ESHG that the EPO should reduce its backlog. Patent examination at EPO typically takes 45 months, but often takes longer for genetic inventions. The scrutiny with which the patent applications are reviewed, and the rules that are followed by the examiners, guarantee that good patents are issued; however, the resulting backlog creates a legal uncertainty in the interim phase. In the case of diagnostics, it leads to situations whereby a diagnostic service has already been well established by the time a (blocking) patent is issued.

One significant way to reduce the influx of patent applications is by narrowing the scope of gene patentability, as suggested in section 3.2. Also, the EPO, together with policy makers, should find a way to adequately deal with applicants abusing their power to make the patent examination and decision process exceedingly long.

\section{(Re)consider the ethical aspect of patenting}

Many disputes between supporters of the patenting system and the public revolve around ethical issues. It is also felt that attorneys, patent applicants and patentees sometimes push the ethical limits of patentability, which damages the perception of the legitimacy of the patenting system as a whole. The ESHG urges EPO to find a way, together with the scientific community and the European institutions, to ensure that a morality issue, when it cannot be addressed under the morality article of EPC, can still be taken into account.

Along the same lines, the ESHG proposes EPO to consider the benefit of having an ethics committee to consider issues of major interest, such as patents applied to genes.

\section{Promote access to information on patents and patent applications}

The EPO already provides free and easily accessible, searchable patent databases (http://ep.espacenet.com/). The nucleotide sequences included in patent applications are available through the Patent_DNA data set of the EMBL nucleotide sequence database. Nevertheless, all possible users should be made better aware of these and other tools. Eventually, it should become equally evident for scientists to search the patent databases as the international scientific literature.

\section{Patient-related issues}

Informed consent documents for participation in research should include information on the fact that the research may lead to patentable inventions.

\section{Patent reform}

The ESHG hopes that the recommendations can be incorporated into the process of patent reform consultation procedures that reportedly already take place at the EPO, the European Commission (EC) and between the two. In any case, the EC and the European Parliament should interact with EPO with respect to the issues raised above, because they are the (only) institutions that may eventually change the policy and law.

\section{Research exemption}

The ESHG finds it necessary to define the scope of the research exemption more explicitly, to make it clear for researchers and clinicians. The ESHG follows the reasoning that diagnostic use (ie the use of a diagnostic test in response to a request from a medical doctor) cannot fall within the research exemption, even if the test is performed within the public health sector and, notably, irrespective of whether money is exchanged or not. It, therefore, strongly advises the genetic laboratories not to rely on the research exemption when offering diagnostic testing.

This notwithstanding, the ESHG urges European and individual national governments to harmonize their policies and practice of research exemption on a European-wide level.

\section{Licensing}

As licenses will be very much necessary to develop new tests, it will be increasingly crucial to facilitate licensing through as efficient a system as possible. The availability of models to secure licenses to sequences and genes, in reasonable practical and financial terms, might encourage scientists to pursue research in different areas, and might encourage users (ie diagnostic laboratories) to seek licenses and pay royalties.

Like health care in general, the delivery of genetic-based diagnostics to the public who needs them is an enterprise that involves human, financial and infrastructural contributions from both the public and the private sector. The IP issues should not further complicate this enterprise, and certainly not hamper it.

\section{Visualise licensing terms}

It would be better if the costs of licensing in the price of the final product would be more transparent. Presently, there are hardly any data available. Parties are therefore encouraged to make licensing agreements public, and to inform 
health-care professionals and the public about the relative contribution of the IP costs to the end product.

\section{Implement guidelines for licensing}

The mechanisms of the market that normally set a fair price on products do not work properly in the case of genetic testing, simply because either there is no way to 'invent around' and put similar products on the market, or the diagnostic laboratories lack the power (ie the patent portfolio) to negotiate reasonable conditions. Policy makers should work on the development of licensing guidelines, and effectively support those that have already been issued by international organisations such as the OECD.

In particular, the ESHG reiterates that license agreements should not provide the licensor with exclusive control over human genetic information. Rights holders should license genetic inventions for health applications, including diagnostic testing, on terms and conditions that seek to ensure the widest public access to, and variety of, products and services based on the inventions. Foundational genetic inventions - and methods for diagnosis - should be licensed so as to be broadly accessible, at a fair and reasonable price. License agreements should not include up-front fees. In any case, licensing practices should not be used to restrict the choice of other products or services by patients and their health-care providers.

The OECD guidelines on licensing should be largely diffused in the research community. The EC would be well advised to further support these guidelines with incentive mechanisms, such as tax incentives.

\section{Promote respect for protected IP}

Mechanisms to improve adherence to licensing guidelines include the promotion of their use and the participation of researchers and clinicians in negotiating and obtaining licenses.

The ESHG urges its members, and the genetic community at large, to respect the patenting and licensing rules once they are acceptable. Research institutes and hospitals should know how to handle licences and patents, and researchers and clinicians should know whom to contact for advice and assistance.

\section{Compulsory licenses}

The ESHG shares the viewpoint that compulsory licenses serve a purpose in keeping patentees from exerting extreme monopoly rights. The models for compulsory licenses, tailored to health care, which have been established now in a few European countries, should be adapted by other European countries into their system, and made consistent at the European level.

\section{Test alternative licensing models}

Patent pools and clearinghouses have been proposed as alternative models for licensing.
A patent pool is an agreement between two or more patent owners to license one or more of their patents to one another, or to license them as a package to third parties who are willing to pay the royalties that are associated with the licence. The clearinghouses refer to any mechanism by which providers and users of goods, services and/or information are matched. The most comprehensive model is the royalty-collection clearinghouse, which is meant to promote technology exchange and, at the same time, cash licence fees from users on behalf of the patent holder, in return for the access to and use of the patented technology.

Several international organisations have previously put forward these models to deal with some of the problems of licensing of genetic inventions; however, to date, these models have been insufficiently studied. More importantly, an international initiative would be necessary to assay their usefulness and validity.

The patent pool/clearinghouse model should be encouraged by governments, by the European institutions and the OECD. One objective could be to establish (a) Europeanwide patent clearinghouse(s) for genetic and biological inventions. A clearinghouse for European research institutes in genetics would facilitate the concentration of gene patent talent and accelerate protection of IP. All stakeholders, including geneticists, insurers, governments, industry and patients, should be involved in this debate.

The ESHG advises the EC to promote an experiment in the field of patent pooling or clearinghouses as a solution to overcome the IP problem and increase access to genetic test and new technology (like biochips). This might be done well through DG Enterprise and DG Research.

Furthermore, the public research funding bodies, starting with DG Research, should encourage the establishment of patent pools within the context of research projects dealing with genes and genetic inventions, and promote the establishment of a platform. One way to do this would be to consider creating fiscal incentives in the biotech sector for the creation of patent clearinghouses for genetic/ biological inventions.

\section{Emphasize ethical aspects of licensing}

The ESHG is calling upon all stakeholders to start the process of developing a code of conduct for partners with patents, covering ethical aspects as well as smooth licensing arrangements. Note that academic institutions are often on both sides of this debate, as patentees on the research and development side, and as users and licensees on the clinical or diagnostic side.

There is a need to consult with all stakeholders. The system should include a mechanism for identifying those companies and institutions that have signed up.

There is a need to set up specific licensing guidelines, especially for IP generated by means of public funding, and to pose incentives for reasonable licensing behaviour. National and international granting agencies should foster 
the development of such guidelines and watch over their application.

\section{Education}

In academic institutions, notably in Europe, publishing comes first, as a source to establish esteem and, through this, as the basis for quality control and fundraising. The need for parallel patenting is too often perceived as a source of delay and distraction. This view impedes the development of a positive attitude towards IP protection and leaves a legacy that has to be overcome in the scientists' future. There is a need to further educate geneticists, other relevant medical communities, and patient organisations in patenting and licensing to help them overcome their apprehension, accept well justified patents and respect IP rights.

The ESHG recommends that a course about IP be a part of a PhD education in the relevant faculties, such as medicine, pharmacy and biology. An education strategy should be elaborated and should take into account the needs of at least three target groups: clinicians, researchers and patient organisations. Clearly written educational material should be available. Specific courses for patient organisations should also be organised.

The academic community is ready to assist in the permanent training of lawyers and EPO examiners, and to exchange views and experiences in the rapidly evolving area of human genetics. The genetic community is well placed to advise about future trends/problems that are linked to particularities of the diagnostics field.

Public health officials should pay attention to and be involved in the consequences surrounding patenting and licensing.

\section{Other (public policy) issues}

In many countries, patent issues are dealt by the Ministry of Justice, even though the consequences affect the Ministry of Health. This dilemma represents the origin of some of the identified problems in this report. Discussion between these ministries is necessary.

A better separation between courts (such as the proposed European Patent Court) and the EPO is desirable, notably considering that EPO is at present not formally accountable to any other body in the EU.

There remains a strong need for harmonisation of the implementation of the EU directive. The main concern lies in its interpretation in different EU countries. Currently, the EC is invited to critically review the implementation of the Biotech Directive 98/44 in each member state and its harmonization with others. In the view of the ESHG, this should optimally be done by an independent committee of experts.
The consequence of the present policy guidelines is that small biotechnology companies are effectively excluded from the market of genetic diagnostics. This poses a potential threat in the breach of existing patents, and subsequently reduces creativity in development, threatening future European benefits in economic development.

There is a tension between property rights in biobanks, subsections thereof, and individual samples therein, and further development based on biobanks. It is worth exploring the declaration of biobanks as common, or community, property. Given the importance of biobanks in discovering small genetic differences in large groups (considered the cause of most common diseases) and the slow process of this discovery, national and European authorities should be urged to consider biobanks not as research projects, but as infrastructures unaffected by short-term profitability expectations. The development of patents in the future will be strongly influenced by the development and outcome of biobank-based research.

Many people would like to see market-free tools (comparable to free software) being developed and promoted whenever possible. This is unlikely to happen if more and more funding agencies evaluate the performance of research laboratories through their number of patents. The ESHG considers this as an unfortunate evolution: research should be primarily driven by intellectual curiosity and the desire to promote knowledge and innovation.

\section{Conclusion}

In the view of the ESHG, the patenting and licensing system will be more easily accepted by the majority of geneticists and by the public, when the specific sensitivities around genetic testing, and of medicine and health care in general, are taken into account in the light of the increasing (and increasingly powerful) diagnostic possibilities coming online. The ESHG herewith proposes several ways to improve the practice and to avoid damaging the quality of care.

There is a need for a constant dialogue between healthcare providers, including geneticists, patent specialists and certainly the EPO, companies, policy makers at the national and European institutions, and patients. The ESHG is willing to contribute to this dialogue.

*Correspondence: Professor G Matthijs, Katholieke Universiteit Leuven, Center for Human Genetics, Herestraat 49, Leuven, Belgium or Professor S Aymé, Hôpital Broussais, INSERM SC11, 102 rue Didot, F-75014 Paris, France.

E-mails: gert.matthijs@uz.kuleuven.ac.be, ayme@orpha.net

\section{Reference}

1 Soini S, Aymé S, Matthijs G, The members of the Public and Professional Policy Committee (PPPC) and Patenting and Licensing Committee (PLC), on behalf of ESHG: Patenting and licensing in genetic testing: ethical, legal and social issues. Eur J Hum Genet 2008; (Suppl i), in press. 